Yuing Farías, T.; Henríquez Flores, R.; Pradanos Salomon, N.; Cortés Villalobos, S. y Curilem Gatica, C. (2019). Efecto hipotensor agudo del ejercicio de oclusión vascular sobre adultos mayores / Cute Hypotensor Effect of the Vascular Occlusion Exercise on Elderly Adults. Revista Internacional de Medicina y Ciencias de la Actividad Física y el Deporte vol. 19 (74) pp. 197-208 Http://cdeporte.rediris.es/revista/revista74/artefecto1027.htm

DOI: http://doi.org/10.15366/rimcafd2019.74.002

\title{
ORIGINAL
}

\section{EFECTO HIPOTENSOR AGUDO DEL EJERCICIO DE OCLUSIÓN VASCULAR SOBRE ADULTOS MAYORES}

\section{ACUTE HYPOTENSOR EFFECT OF THE VASCULAR OCCLUSION EXERCISE ON ELDERLY ADULTS}

\author{
Yuing Farías, T.1; Henríquez Flores, R.1; Pradanos Salomon, N. ${ }^{1}$; Cortés \\ Villalobos, S. ${ }^{1}$ y Curilem Gatica, C. ${ }^{1}$
}

1 Escuela de Kinesiología, Facultad de Salud, Universidad Santo Tomás, Chile. tuillangyuing@santotomas.cl,ricardohenriquezfl@santotomas.cl, ninoska.pradanos@gmail.com, sebac-16@hotmail.com, c.curilem.g@gmail.com

Código UNESCO / UNESCO Code: 321311 Fisioterapia / Physiotherapy Clasificación Consejo de Europa / Classification Council of Europe: 14 Fisioterapia y rehabilitación / Physiotherapy and rehabilitation

Recibido 18 de julio de 2017 Received July 18, 2017

Aceptado 15 de enero de 2019 Accepted January 15, 2019

\section{RESUMEN}

Objetivo: El objetivo de este estudio fue evaluar la respuesta cardíaca aguda después de realizar una sesión de ejercicio con oclusión vascular en adultos mayores (AM). Sujetos y método: 22 participantes voluntarios, fueron sometidos al protocolo experimental de ejercicio dinámico de prensión manual con un $30 \%$ de presión de oclusión (PO), después de registrar los valores basales y en reposo de presión arterial sistólica (PAS), presión arterial diastólica (PAD) y frecuencia cardíaca (FC). Evaluadas en condiciones basales y entre los 5 y 30 minutos post-oclusión. Resultados: Al finalizar el protocolo se observó una reducción significativa en la PAS y FC a los $15^{\prime}, 20^{\prime}$ y $25^{\prime}(p<0,05)$ y para la PAD a los 10' y 20' $(p<0,05)$. Conclusión: El efecto agudo del ejercicio con oclusión vascular genera una reducción significativa de PAS, PAD y FC hasta 25 minutos post-oclusión, en los AM analizados.

PALABRAS CLAVES: Hipertensión; Hipotensión post ejercicio; Oclusión terapéutica. 


\section{ABSTRACT}

The objective of this study was to evaluate the acute cardiac response after performing an exercise session with vascular occlusion in elderly adults (EA). Twenty-two volunteer participants underwent the experimental protocol of dynamic manual grip exercise with $30 \%$ occlusion pressure (OP), after recording the baseline values and resting systolic blood pressure (SBP), diastolic blood pressure (DBP) and heart rate (HR). All measures were taken in basal conditions and between 5 and 30 minutes' post-occlusion. At the end of the protocol, there was a significant reduction in SBP and HR at 15 ', 20' and 25 ' $(p<0.05)$ and for DBP at $10^{\prime}$ and 20 ' $(p<0.05)$.). The acute effect of exercise with vascular occlusion generates a significant reduction of SBP, Basal Diastolic Pressure (BDP) and HR up to 25 minutes' post-occlusion, in the EA analyzed.

KEYWORDS: Hypertension; Post-Exercise Hypotension; Therapeutic Occlusion.

\section{INTRODUCCIÓN}

La intolerancia al ejercicio es una característica común de múltiples enfermedades crónicas no transmisibles, incluyendo la hipertensión arterial (HTA). Se han postulado varios mecanismos, como anormalidades en el metabolismo muscular, alteraciones en la absorción y utilización del oxígeno muscular y especialmente restricciones del flujo sanguíneo muscular, para explicar esta baja tolerancia al esfuerzo ${ }^{(1)}$.

La HTA es una enfermedad y un factor de riesgo cardiovascular, se caracteriza por un aumento elevado, progresivo y mantenido en el tiempo, de la presión arterial sistólica (PAS), diastólica (PAD) o ambas. La HTA es más frecuente en los adultos mayores (AM) (2), producto de cambios que ocurren con el envejecimiento ${ }^{(3,4)}$. En la HTA se puede alterar la regulación del tono vascular, generando un predominio de la vasoconstricción sobre la vasodilatación, lo que se traduce en un aumento de la resistencia vascular periférica (RVP) y que puede ir acompañada de restricción del flujo sanguíneo periférico ${ }^{(5)}$.

Varios factores han sido involucrados en la RVP, tales como: aumento del tono vasomotor, irregularidad del lumen de los vasos y procesos de inflamación de bajo grado; que aumentan la resistencia al flujo de sangre. Sin embargo, estudios realizados en diversos modelos sugieren que estos factores no son suficientes para explicar el aumento en la RVP, por lo que han sido evaluados otros factores que están íntimamente relacionados, como propiedades del flujo sanguíneo o la restricción del flujo por una capa macromolecular sobre la superficie endotelial ${ }^{(6)}$.

El ejercicio regular es una intervención bien establecida para la prevención y tratamiento de la HTA ${ }^{(7)}$. Las personas pueden beneficiarse clínicamente de hipotensión post-ejercicio (HPE) ${ }^{(8,9)}$ que tiene una mayor duración cuando se realiza de $2-3$ veces por semana ${ }^{(10,11)}$. Los efectos 
hipotensores del ejercicio parecen estar inducidos principalmente por una mejora en la sensibilidad barorefleja ${ }^{(12)}$, supresión de actividad simpática ${ }^{(9)}$, incremento en el gasto cardíaco ${ }^{(13)}$ y la liberación de vasodilatadores ${ }^{(14)}$.

Por lo mencionado hasta ahora, es que se ha indagado en metodologías que reduzcan la RVP, con la consecuente reducción en la presión arterial (PA), así como también generen una hiperemia durante el ejercicio, esta respuesta sigue siendo un punto de controversia. Algunos mecanismos involucrados en esta respuesta son: regulación neural, respuesta endotelial y regulación de la función cardíaca. Dentro de las metodologías más utilizadas se menciona al entrenamiento intervalado de alta intensidad $(\mathrm{HI})$, el cual ha demostrado muy buenos efectos en hipertrofia muscular y reducción de la PA de manera aguda (post ejercicio) y crónica con el entrenamiento planificado ${ }^{(15)}$. En AM se ha demostrado que el entrenamiento de HI presenta altos riesgos de lesión, dificultando la adherencia de los sujetos ${ }^{(16)}$. En este contexto, la oclusión vascular ha sido destacada por su seguridad y los efectos similares a los obtenidos por el entrenamiento de $\mathrm{HI}$, pero a intensidades más bajas y con menores riesgos de lesión ${ }^{(17)}$. De la oclusión vascular se han identificado diferentes usos y beneficios, de los cuales los más estudiados son el aumento de la fuerza e hipertrofia muscular. Estos resultados generarían una mayor vasodilatación periférica y un aumento del metabolismo muscular $(18,19)$. Estos efectos permiten optar por el entrenamiento con oclusión vascular, como un método seguro, aplicable en AM y con efectos similares a la $\mathrm{HI}$.

Por otro lado, en un estudio de Neto ${ }^{(20)}$ uno de los efectos observados es la disminución de la PA en sujetos normotensos luego de la aplicación del ejercicio oclusivo pasando de $119 \pm 8,9 \mathrm{mmHg}$ de presión arterial media en reposo a $113 \pm 11,1 \mathrm{mmHg}$, luego de 60 min posterior al entrenamiento ${ }^{(20)}$.

En base a estos antecedentes, el entrenamiento de oclusión vascular podría ser una herramienta útil en el trabajo con poblaciones de riesgo, como lo son los AM. En normotensos se sabe que puede controlar de manera aguda la $\mathrm{PA}$, pero se desconoce cuales podrían ser sus efectos en AM con presiones arteriales elevadas.

Por lo tanto, el propósito de este estudio es evaluar los efectos agudos posterior a la realización de una serie de ejercicio bajo oclusión vascular en AM.

\section{MATERIAL Y MÉTODOS}

Se seleccionó una muestra a conveniencia, donde se invitó a los participantes mediante una carta dirigida a la agrupación de AM "Luz de luna" de las comunas de Los Vilos, IV región Chile, la cual fue acogida por un total 22 sujetos de ambos géneros. Todos los participantes de esta investigación leyeron y firmaron el consentimiento informado antes de participar en el estudio. El proceso de selección y tratamiento de los sujetos fue aprobado por el Comité de Ética centro/norte de la Universidad Santo Tomás. Se seleccionó a los sujetos que cumplieran con criterios de inclusión referentes a que fueran $\geq$ a 65 años, con residencia en la comuna de Los Vilos, IV región-Chile y que hubiesen firmado 
el consentimiento informado. Los criterios de exclusión para la muestra fueron: que presente un grado de dependencia que limiten la práctica de ejercicio, presentar patologías cardiovasculares, presentar antecedentes trombóticos o evento cardiovascular en los últimos 2 años y que haya realizado ejercicio vigoroso durante las últimas 24 horas previas a la recolección de datos (Fig 1).

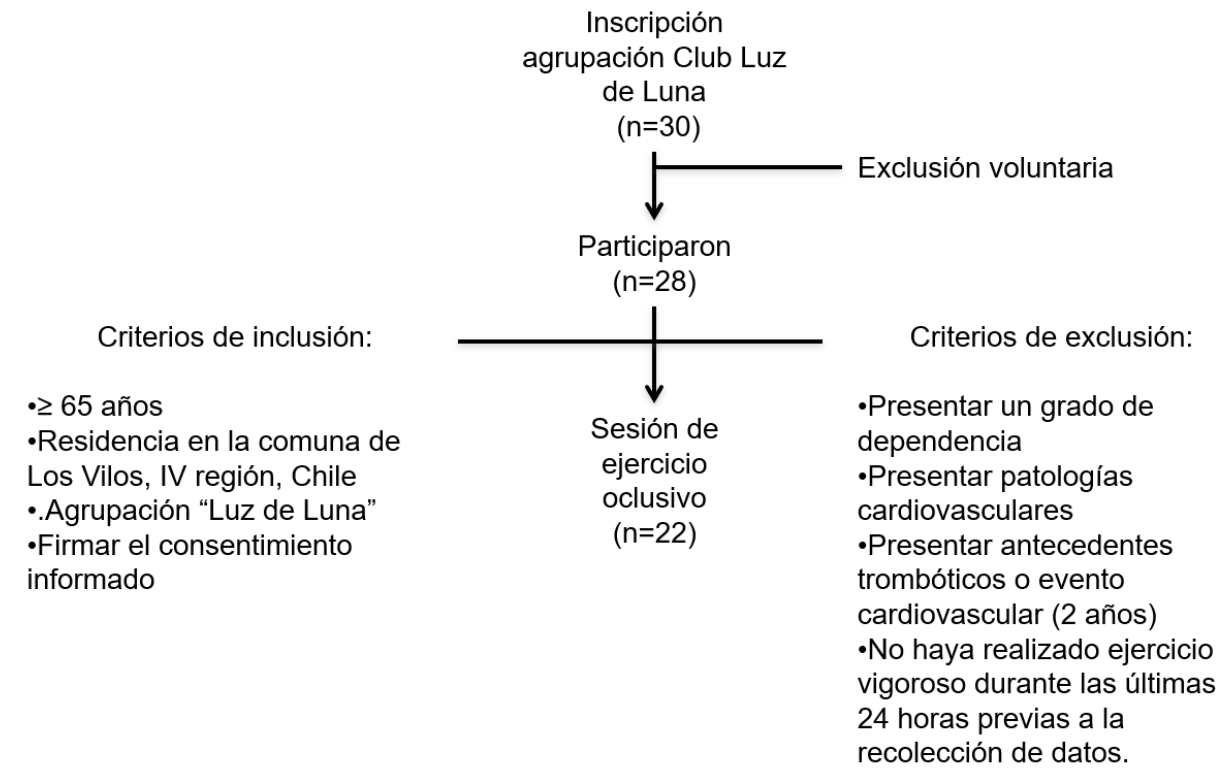

Fig. 1. Diseño del estudio. Treinta sujetos respondieron a la invitación a participar en este estudio. Dos sujetos no se presentaron a la sesión y seis no cumplieron los criterios de inclusión / exclusión. Veintidós sujetos realizaron la sesión de ejercicio.

\section{Procedimientos del estudio}

A los sujetos seleccionados se les recolectó su historia clínica y sus medidas del peso corporal, estatura e índice de masa corporal (IMC).

\section{Protocolo de ejercicio oclusivo}

La sesión tuvo duración de 40 minutos, se inicia tomando las presiones pre-ejercicio, la Presión arterial basal (PAB) se registra posterior a 5 minutos de reposo en posición supina y la Presión arterial en reposo (PAR) será posterior a 3 minutos en la posición sedente, la presión sedente se utilizará para determinar la presión de oclusión $(\mathrm{PO})$ calculada como $\mathrm{PO}=\left(\mathrm{PAS}^{\star} 0,3\right)+\mathrm{PAS}$.

Posteriormente se realizó 1 minuto de ejercicio dinámico con el miembro dominante, donde el segmento de antebrazo estará apoyado sobre una mesa, con la articulación de codo en un ángulo de $90^{\circ}$. Una vez aplicada la $\mathrm{PO}$ se le solicitó al paciente un minuto de prensión manual o hasta el fallo muscular.

Posterior a esto se evaluó la PA post-ejercicio a los 5, 10, 15, 20, 25 y 30 minutos post-oclusión. Las mediciones de la PA se tomaron de acuerdo a lo descrito por la guía clínica de HTA primaria o esencial en personas de 15 años y más. 


\section{Análisis estadístico}

Para la realización de este análisis se utilizó estadística descriptiva, utilizando medida de tendencia central a través del promedio o media aritmética y de dispersión mediante el error estándar de la media. Para comprobar la distribución normal, se utilizó la prueba de Shapiro Wilk. Para la estadística inferencial, los datos se procesaron con prueba de t-student pareado para grupos que tuviesen una distribución normal y se utilizó la prueba de Wilcoxon para los que no tuviesen una distribución normal. Los valores se muestran como media \pm DE y la significancia estadística se definió con un $p<0,05$.

\section{RESULTADOS}

Un total de 22 participantes, 17 mujeres y 5 hombres, completaron la totalidad del protocolo. En la tabla 1 se resumen las características demográficas de los participantes del estudio. El $53 \%$ de las mujeres y el $60 \%$ de los hombres se encuentran en la categoría de sobrepeso para su rango de edad (21). Además, el $11,76 \%$ de las mujeres y el $20 \%$ de los hombres tenían PAS basales sobre el rango normal para su edad, al registrar la PAD, el 5,9\% de las mujeres y el $20 \%$ de los hombres, se encontraban sobre el valor normal para su edad. De la misma forma, al medir su PAS y PAD en reposo, un porcentaje no menor de mujeres y hombres se encontraban por fuera del rango de normalidad (PAS=17,64\%, 40\%; $\mathrm{PAD}=17,64 \%, 0 \%$, mujeres y hombres respectivamente).

Los cambios que se producen en la PAS y PAD antes y después de la sesión de ejercicio con oclusión vascular se muestran en la figura 2 . Existe un aumento significativo en la PAS al pasar del basal a la posición sedente en reposo $(p<0,05)$ y en la PAD $(p<0,001)$. Este aumento persiste a los 5 minutos después de realizar la sesión de ejercicio con oclusión, tanto para la PAS como para la PAD $(p<0,01)$. A los 10', 15' y 20 ' hay una reducción significativa con respecto al valor basal para la $\operatorname{PAD}(p<0,05 ; p<0,01 ; p<0,05$ respectivamente).

Por último, hubo una reducción significativa en la PAS con respecto a la PAR a los 15', 20' y 25' ( $p<0,05)$ y para la PAD a los 10' y $20^{\prime}(p<0,05)$. 
Tabla 1. Características demográficas de la muestra $(n=22)$.

Promedio \pm Intervalo de Mínimo Maximo
Desviación Confianza estándar $\quad(95 \%)$

\begin{tabular}{ccccc}
\hline Edad (años) & $71,86 \pm 4,06$ & $(68,5-74,5)$ & Min 65 & Max 81 \\
\hline Peso (Kg) & $74,45 \pm 12,78$ & $(64,5-85,5)$ & Min 49 & Max 97 \\
\hline Talla (m) & $1,573 \pm 0,1$ & $(1,52-1,63)$ & Min 1,34 & Max 1,84 \\
\hline IMC (Kg/m $\left.{ }^{2}\right)$ & $29,95 \pm 3,4$ & $(27,5-32)$ & Min 24 & Max 36 \\
\hline PASB (mmHg) & $131,4 \pm 8,4$ & $(124-138,5)$ & Min 116 & Max 149 \\
\hline PADB (mmHg) & $72,86 \pm 10,95$ & $(61-79)$ & Min 58 & Max 96 \\
\hline PASR (mmHg) & $135,1 \pm 10,59$ & $(126,5-141,5)$ & Min 119 & Max 163 \\
\hline PADR (mmHg) & $79,05 \pm 7,98$ & $(61-85,5)$ & Min 65 & Max 95
\end{tabular}

IMC, índice de masa corporal; PASB, presión arterial sistólica basal; PADB, presión arterial diastólica basal; PASR, presión arterial sistólica reposo; PADR, presión arterial diastólica reposo; IC, intervalo de confianza; Min, valor mínimo; Máximo, valor máximo.

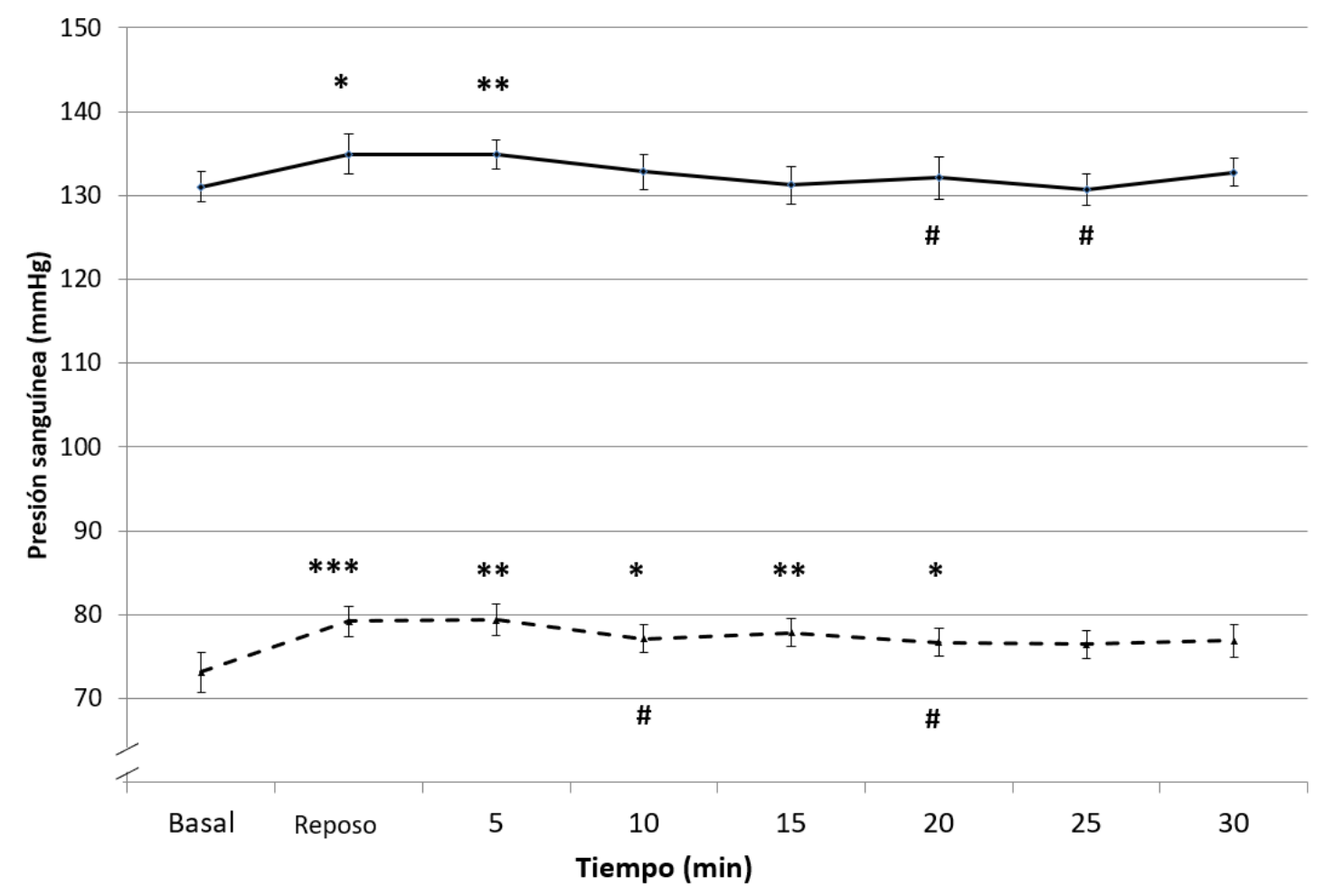

Fig. 2: Cambios en la presión arterial sistólica y presión arterial diastólica, antes y después de la sesión de ejercicio con oclusión vascular. PAS: presión arterial sistólica (Línea continua), PAD: presión arterial diastólica (Línea discontinua). *: Diferencia significativa con respecto al valor basal, \#: diferencia significativa con respecto al reposo. ${ }^{*}=p<0,05 ;{ }^{* *}=p<0,01 ;{ }^{* *} ; p<0,001$; $\#=p<0,05$

Con respecto a los cambios que se producen en la FC, estos se muestran en la figura 3. Existe un aumento significativo entre la posición basal y la de reposo y que se mantiene elevada a los 5' y 10' después de realizar el ejercicio 
con oclusión vascular $(p<0,01$ para posición de reposo y 5'; $p<0,05)$. Por otro lado, se muestra una reducción significativa $(p<0,05)$ a los 10 ' posteriores a la ejecución de la sesión de ejercicio con oclusión vascular y muestra una reducción significativa cada vez mayor y que se extiende hasta los 30' de finalizado el ejercicio ( $p<0,05$ a los $15^{\prime}, 20$ ', 25 ' y 30 ' respectivamente).

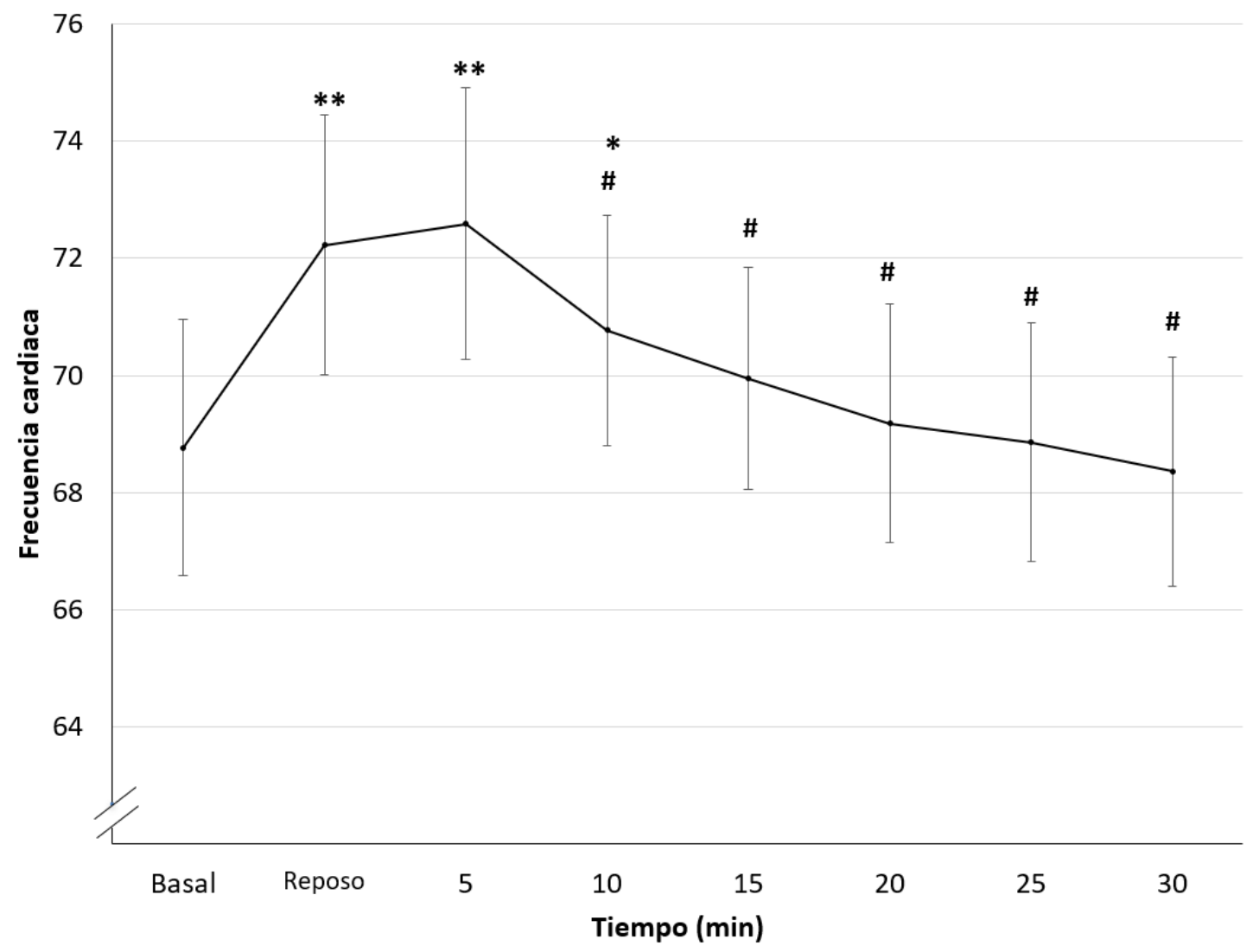

Fig. 3: Cambios en la frecuencia cardíaca, antes y después de la sesión de ejercicio con oclusión vascular. *: Diferencia significativa con respecto al valor basal, \#: diferencia significativa con respecto al reposo. ${ }^{*}=p<0,05 ;{ }^{* *}=p<0,01 ; \#=p<0,05$.

\section{DISCUSIÓN}

El objetivo del presente fue evaluar la respuesta aguda sobre la PA y FC después de realizar una sesión de ejercicio con oclusión vascular en AM. En este contexto, los resultados obtenidos en la presente investigación ponen de manifiesto reducciones significativas de la PAS $(p<0,05)$ a los $15^{\prime}, 20^{\prime}$ y $25^{\prime} ;$ y de la PAD a los 10', 15' y 20' ( $<<0,05 ; p<0,01, p<0,05$ respectivamente), posteriores a la realización de una sesión de ejercicio con oclusión vascular.

Estos datos confirman que la PA puede disminuir hasta 25' minutos después del ejercicio y la prescripción de esta modalidad, con una breve duración y alta intensidad percibida por la oclusión, es segura para los AM (cambios en la presión no eran riesgosos). Si bien es cierto se generaron reducciones significativas en la PAS y PAD una vez finalizado el ejercicio con oclusión vascular, antes de la sesión de ejercicio, los niveles de PA estaban adecuadamente controlados según las directrices internacionales ${ }^{(22) .}$ 
En nuestros resultados existe un aumento en la PAS y PAD al pasar de la posición basal a la de reposo y este cambio persiste 5' posteriores a realizar el ejercicio con oclusión vascular, cabe mencionar que la menor masa muscular y menor capilaridad de los miembros superiores, en conjunto con el aumento de la resistencia al flujo sanguíneo impuesto por la oclusión vascular, conlleva a un aumento en la PA. Este efecto es similar a lo que ocurre al realizar las últimas repeticiones de una serie de ejercicios aumentando la PA intraesfuerzo ${ }^{(26)}$. Se ha postulado que la realización de ejercicio de resistencia de grandes grupos musculares genera una HPE ${ }^{(28,229)}$ tanto en personas normo tensas como en personas hipertensas y que el mayor efecto de reducción de PA se daría en estas últimas. Además de ello, se comparó el efecto de realizar este tipo de ejercicios 2 a 3 veces por semana, en donde no se encontraron diferencias significativas en las reducciones de los valores de PA según el número de sesiones de entrenamiento semanal.

Un estudio realizado el año $2010{ }^{(21)}$, comparó la HPE de un ejercicio dinámico con oclusión vascular en AM hipertensos sedentarios versus los entrenados. En él se encontró que en ambos grupos redujeron significativamente la PA, pero el mayor cambio se generó en el grupo sedentario. Esto indicaría que es posible realizar este tipo de ejercicio en pacientes AM con HTA y que podría generar un cambio importante en los valores de PA con o sin el complemento del tratamiento farmacológico.

Tanto los tratamientos farmacológicos como el ejercicio físico contribuyen a reducir la PA y los riesgos asociados a su aumento. Es bien conocido que los beneficios del ejercicio físico se manifiestan a largo plazo y cuando existen adaptaciones crónicas al ejercicio y estos efectos han estado asociados principalmente a diferentes modalidades de ejercicio aeróbico ${ }^{(23,24,25)}$. De esta misma forma, en un estudio se comprobó sólo una reducción significativa de la PAS a los 60' de realizar el protocolo de alta intensidad en prensa de piernas. Por otro lado, al realizar un ejercicio con oclusión vascular, al 20\% de 1 repetición máxima (RM) y con una oclusión de $200 \mathrm{~mm} \mathrm{Hg}$ que resultó sin cambios significativos a los $30^{\prime}$ y $60^{\prime}$ después de realizar el ejercicio. Si bien es cierto, no hubo diferencias significativas, se consideró que la PO no fue lo suficientemente intensa para desarrollar una HPE y que existiría una relación directa entre aumento de la resistencia a vencer y la respuesta hipotensiva, siendo a mayor oclusión, mayor disminución en las variables de PA.

Aunque se han postulado algunos mecanismos fisiológicos para explicar la HPE, aún no se ha llegado a un consenso al respecto. La reducción de la actividad autonómica simpática es una de las hipótesis identificadas como responsables de la HPE, esta a su vez estaría asociada con vasodilatadores que conducen a una reducción en la RVP y, en consecuencia, disminución de la PA (30).

Otro aspecto para mencionar con respecto a la posible reducción de la actividad simpática es la reducción de la FC. Nuestros resultados muestran una reducción significativa después de realizar la sesión de ejercicio con oclusión vascular, con una clara tendencia hacia la disminución y que se extiende hasta 
los 30'. Estas dos características de reducción de la PA y FC, pueden deberse a una reducción de la actividad autonómica simpática o a su vez debido a una mejora en la perfusión tisular, lo que traería como consecuencia una mayor eficiencia en el trabajo cardíaco ${ }^{(31)}$.

Todos los efectos mencionados hasta ahora son producto de períodos de entrenamiento, vale decir, la respuesta crónica de respuesta hipotensora frente a ejercicios de resistencia o de oclusión vascular, pero no se había investigado la respuesta aguda frente al ejercicio de oclusión vascular. Esta pareciera ser similar a la respuesta crónica, pero limitada en el tiempo, no extendiéndose más allá de los 30', pero al considerar la respuesta frente a una sola sesión de ejercicio de oclusión vascular, además de lo documentado como respuesta crónica, sentaría las bases para la aplicación de este tipo de entrenamiento en el manejo y control no farmacológico de la HTA.

Los datos reportados en este estudio, junto con la evidencia actual respecto a la utilidad clínica que podría tener este tipo de metodología en el manejo y control de la HTA, aún quedan muchos aspectos fisiológicos por explorar y aclarar, sin embargo, se ha postulado que los ejercicios con grandes grupos musculares, así como también la PO utilizada, influyen en la intensidad y duración de la respuesta post ejercicio.

\section{CONCLUSIÓN}

El efecto agudo de un ejercicio dinámico con oclusión vascular genera una disminución significativa en la PA y FC durante los primeros 25 minutos post ejercicio en los AM estudiados, por lo que podría ser una opción de tratamiento no farmacológico de la HTA.

\section{AGRADECIMIENTOS}

A la Dirección de Investigación y Post grado de la Universidad Santo Tomás por su constante apoyo en el desarrollo de proyectos de investigación y difusión científica.

\section{REFERENCIAS BIBLIOGRÁFICAS}

1. - Lash J. Regulation of skeletal muscle blood flow during contractions. Proc Soc Exp Biol Med. 1996; 211:218-35.https://doi.org/10.3181/00379727211-43965. PMid:8633102.

2. -Picon, R.; Fuchs, F.; Moreira, L.; Fuchs, S. Prevalence of hypertension among elderly persons in urban Brazil: a systematic review with metaanalysis. Am J Hypertens. 2013; 26 (4):541-8. https://doi.org/10.1093/ajh/hps076. PMid:23467209.

3. -Safar, M.; Benetos, A. Factors influencing arterial stiffness in systolic hypertension in the elderly: role of sodium and therenin-angiotensin system. Am J Hypertens. 2003; 16 (3):249-58. https://doi.org/10.1016/S0895-7061(02)03259-4.

4. -Zieman, S.; Melenovsky, V.; Kass, D. Mechanisms, pathophysiology, and therapy of arterial stiffness. Arterioscler Thromb Vasc Biol. 2005; 25 
(5):932-43.

https://doi.org/10.1161/01.ATV.0000160548.78317.29. PMid:15731494.

5.- Alvarez, C. 2013. Efectos de una sesión de ejercicio aeróbico en la presión arterial de niños, adolescentes y adultos sanos. Rev Med Chile. 2013; 141: 1363-1370. https://doi.org/10.4067/S0034-98872013001100001. PMid:24718461.

6.- Risler, N.; Miatello, M.; \& Cruzado, M. 2002. La pared vascular en la hipertensión arterial. Rev Fed Arg Cardiol. 2002; 31: 315-320.

7. Cornelissen, V.; Smart, N. Exercise training for blood pressure: a systematic review and meta-analysis. J Am Heart Assoc. 2013; 2(1): e004473. https://doi.org/10.1161/JAHA.112.004473. PMid:23525435 PMCid:PMC3603230.

8. Cunha, R.; Jardim, P. Subacute blood pressure behavior in elderly hypertensive women after resistance exercise session. J Sports Med Phys Fitness. 2012; 52 (2):175-80.

9. Wong, M. L.; Formiga, M. F.; Owens, J.; Asken, T.; Cahalin, L. P. Safety of Blood Flow Restricted Exercise in Hypertension: A Meta-Analysis and Systematic Review With Potential Applications in Orthopedic Care. Tech Orthop. 2018; 33(2), 80-8. https://doi.org/10.1097/BTO.0000000000000288

10. Kiyonaga, A.; Arakawa, K.; Tanaka, H.; Shindo, M. Blood pressure and hormonal responses to aerobic exercise. Hypertension. 1985; 7 (1):12531. https://doi.org/10.1161/01.HYP.7.1.125. PMid:2984115

11. Seals,D.; Reiling, M. Effect of regular exercise on 24-hour arterial pressure in older hypertensive humans. Hypertension 1991; 18 (5):583-92. https://doi.org/10.1161/01.HYP.18.5.583. PMid:1937660.

12. Chen, C.; Bonham, A. Post exercise hypotension: central mechanisms. Exerc Sport Sci Rev. 2010; 38(3):122-7. https://doi.org/10.1097/JES.0b013e3181e372b5 PMid:20577060 PMCid:PMC2936915.

13. Luttrell, M.; Halliwill, J. Recovery from exerciseF: vulnerable state, window of opportunity, or crystal ball? Front Physiol. 2015; 6: 204. https://doi.org/10.3389/fphys.2015.00204. PMid:26257656 PMCid:PMC4510411.

14. Santana, H.; Moreira, S.; Asano, R.; et al. Exercise intensitymodulates nitric oxide and blood pressure responses in hypertensive older women. Aging Clin Exp Res. 2013; 25 (1):43-8. https://doi.org/10.1007/s40520-0130017-x. PMid:23740632.

15. Wilson, C.; Perkin, OJ.; McGuigan, MP.; et al. The effect of age on technique variability and outcome variability during a leg press. PLoS One. 2016;11:e0163764 https://doi.org/10.1371/journal.pone.0163764. PMid:27701431 PMCid:PMC5049752.

16. Burton, E.; Hill, AM.; Pettigrew, S.; et al. Why do seniors leave resistance training programs? Clin Interv Aging. 2017; 12:585-592. https://doi.org/10.2147/CIA.S128324 PMid:28392682 PMCid:PMC5375632.

17. Ozaki, H.; Miyachi, M.; Nakajima, T.; et al. Effects of 10 weeks walk training with leg blood flow reduction on carotid arterial compliance and muscle 
size in the elderly adults. Angiology. $2011 ; 62: 81-86$.. https://doi.org/10.1177/0003319710375942. PMid:20682613.

18.- Ohta, H.; Kurosawa, H.; Ikeda, H.; Iwase, Y. et al. Low load resistance muscular training with moderate restriction of blood flow after anterior cruciate ligament reconstruction. Acta Orthop Scand. 2003 ; 74(1): 62-8. https://doi.org/10.1080/00016470310013680. PMid:12635796.

19.- Hernández, M. \& Herrero, A. Respuestas y adaptaciones al entrenamient oclusivo de baja intensidad. EF Deportes, Revista Digital. 2012.; 16, № 164.

20.- Neto, G.; Sousa, M.; Costa, P.; Salles, B.; Novaes, G; \& Novaes, J. Hypotensive effects of resistance exercises with blood flow restriction. J Strength Cond Res. 2015; 29(4):1064-70. https://doi.org/10.1519/JSC.0000000000000734. PMid:25330083.

21.- Tercer Estudio para el Examen de la Salud y la Nutrición (NHANES III) EN: J AM Diet. Assoc. 2000; 100: 59-66.

22. Chobanian, A.; Bakris, G.; Black; H. et al. The seventh report of the joint national committee on prevention, detection, evaluation, and treatment of high blood pressure: the JNC 7 report. JAMA. 2003; 289(19):2560-72. https://doi.org/10.1001/jama.289.19.2560

PMid:12748199.

23. Lemes, Í.; Ferreira, P.; Linares, S. et al. Br J Sports Med 2016; 50:14381442. https://doi.org/10.1136/bjsports-2015-094715. PMid:26964146 PMCid:PMC5136729.

24. Farahani, A.; Mansournia, M.; Asheri, H. et al. The effects of a 10- week water aerobic exercise on the resting blood pressure in patients with essential hypertension. Asian J Sports Med. 2011;32(3):159-67. https://doi.org/10.5812/asjsm.34854.

25.-Rossow, L.; Fahs, C.; Sherk, V.; Seo, D.; Bemben, D.\& Bemben, M. The effect of acute blood-flow-restricted resistance exercise on post exercise blood pressure. Clin Physiol Funct Imaging. 2011; 31(6), 429-434. https://doi.org/10.1111/i.1475-097X.2011.01038.x. PMid:21981453.

26. Manini, T.; Clark, B. Blood flow restricted exercise and skeletal muscle health. Exerc Sport Sci Rev. . 2009; 37(2), 78-85. https://doi.org/10.1097/JES.0b013e31819c2e5c. PMid:19305199.

27.- Costa, J.;Gerage, A.; Gonçalves, C.; Pina, F.; Polito, M. Influencia del estado de entrenamiento en el comportamiento de la presión arterial después de una sesión de entrenamiento con pesas en ancianos hipertensos. Rev Bras Med Esporte. 2010; 16(2):103-106. https://doi.org/10.1590/S1517-86922010000200005.

28.- Mediano, M.; Paravidino, V.; Simão, R.; Pontes, F.; Polito, M. Comportamiento de la presión arterial subaguda después de un entrenamiento de fuerza en hipertensos controlada. Rev Bras Med Esporte. 2005; 11(6):337-340. https://doi.org/10.1590/S151786922005000600006.

29.-Simão, R.; Santos, E. Comportamiento de la presión arterial después de una sesión de ejercicios de resistencia. Fitness \& Performance Journal. 2005; 4(4): 227 - 231. https://doi.org/10.3900/fpj.4.4.227.s.

30.Brownley, K.; Hinderliter, A.; West, S.; Girdler, S.; Sherwood, A.; Light, K. Sympathoadrenergic mechanisms in reduced hemodynamic stress 
responses after exercise. Med Sci Sports Exerc. 2003; 35(6): 978-86.

https://doi.org/10.1249/01.MSS.0000069335.12756.1B. PMid:12783046.

31.- Rondon, M.; Alves, M.; Braga, A.; Teixeira, O.; Barretto, A.; Krieger, E. e Negrão, C. Post-exercise blood pressure reduction in elderly hypertensive patients. J Am Coll Cardiol. 2002; 39:676-82. https://doi.org/10.1016/S0735-1097(01)01789-2.

Número de citas totales / Total references: 31 (100\%)

Número de citas propias de la revista / Journal's own references: 1 (3,22\%)

Rev.int.med.cienc.act.fís.deporte - vol. 19 - número 74 - ISSN: 1577-0354 